\title{
Biomechanik des Meniskus
}

\author{
L. Dürselen, M. Freutel
}

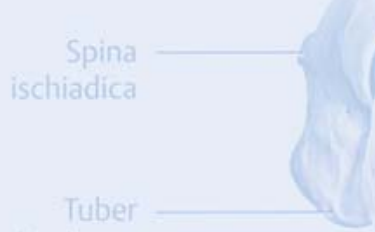

chiadicun

Die Hauptfunktion der knorpeligen Menisken des Kniegelenks ist die Reduzierung des Druckes auf den femoralen und tibialen Gelenkflächen. Die biomechanische Funktion der Menisken wird durch ihre spezielle Anatomie und ihren ultrastrukturellen Aufbau gewährleistet. Daneben kommt auch den meniskotibialen Bändern eine entscheidende Bedeutung zu.

Meniskusverletzungen machen häufig Teil- oder - seltener die Totalresektion eines Meniskus erforderlich. Dies führt zur signifikanten Erhöhung des Kontaktdruckes und in der Folge nicht selten zu Gelenkarthrose.
Um die Funktionsweise des Meniskus und die Auswirkungen von Verletzungen und operativen Eingriffen auf die Kniegelenkmechanik zu verstehen, ist ein Grundverständnis des anatomischen Aufbaus und der biomechanischen Funktionsweise des Meniskus sowie seiner Materialeigenschaften erforderlich. Dieser Artikel soll dem Orthopäden und Unfallchirurgen einen Überblick über die biomechanische Funktionsweise des Meniskus und seiner operativen Behandlung geben.

\section{Einleitung}

Meniskusläsionen werden durch traumatische Ereignisse oder degenerative Prozesse verursacht und sind meist von Schmerz, Gelenkblockierung und Ergussbildung begleitet. Sie sind deshalb der häufigste Grund für einen heute in der Regel arthroskopisch durchgeführten operativen Eingriff am Kniegelenk. Dabei stellt die partielle Meniskektomie den weitaus größten Anteil aller Meniskusoperationen.

Die teilweise oder vollständige Entfernung des Meniskus wirkt sich jedoch in hohem Maße nachteilig auf die Kraftübertragung im Kniegelenk in Form einer Erhöhung der Belastung der knorpeligen Gelenkflächen aus und kann langfristig zu einer Gonarthrose führen. Aus diesem Grund wird heutzutage eine möglichst sparsame Meniskusresektion oder, wenn möglich, die Rekonstruktion bzw. der Ersatz zerstörten Meniskusgewebes angestrebt.

\section{Abkürzungen}

E-Modul

Elastizitätsmodul

ISAKOS International Society of Arthroscopy, Knee Surgery and Orthopaedic Sports Medicine

\section{Anatomie}

\section{Meniskus}

Der laterale und mediale Meniskus des Kniegelenks befinden sich zwischen den artikulierenden Flächen der Femurkondylen und des Tibiaplateaus (Abb. 1).

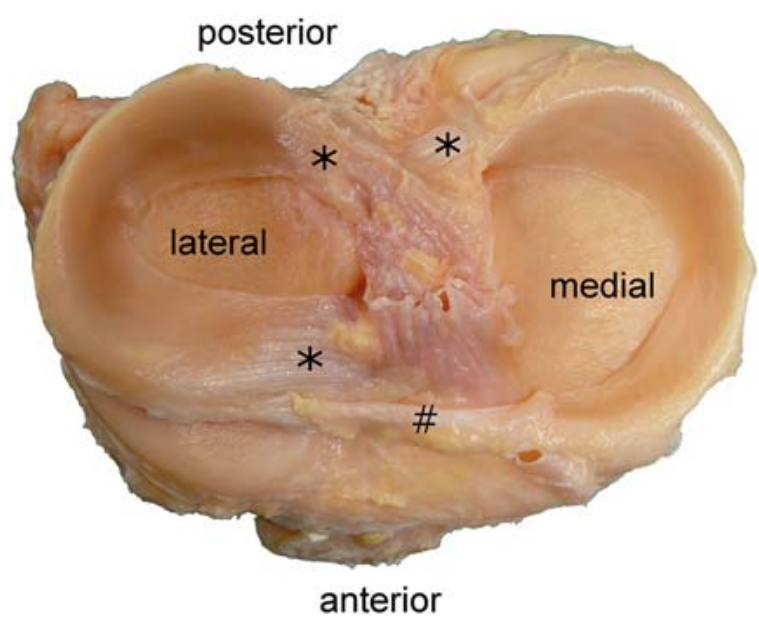

Abb. 1 - Ansicht von proximal eines rechten Tibiaplateaus mit medialem und lateralem Meniskus und den jeweiligen anterioren und posterioren Verankerungsligamenten (* [das anteromediale Verankerungsligament ist verdeckt]). Das Lig. transversum (\#) verbindet die Vorderhörner der beiden Menisken. 
Von anterior nach posterior wird der jeweilige Meniskus unterschieden in

- Vorderhorn,

- Pars intermedia und

- Hinterhorn.

Beide Menisken weisen eine halbmondförmige Form mit einem keilförmigen Querschnitt auf. Der periphere Rand des medialen Meniskus ist mit dem Innenband verwachsen und umlaufend mit der Gelenkkapsel, was zu einer geringeren Beweglichkeit im Vergleich zum lateralen Meniskus während der Flexion und Extension führt. Die Form des Meniskus zusammen mit seinem mikrostrukturellen Aufbau definieren die biomechanischen Eigenschaften und die Funktion des Meniskus.

Der fibrokartilaginäre Meniskus wird gewöhnlich durch seine zwei Phasen (biphasisch) charakterisiert, bestehend aus

- interstitieller Flüssigkeit (60-70\%),

- einer porösen Festkörpermatrix mit Kollagenfasern hauptsächlich vom Typ I (15-25\%) und Proteoglykanen $(1-2 \%)[1]$.

Petersen u. Tillmann [2] wiesen mit rasterelektronenmikroskopischen Aufnahmen einen dreischichtigen Aufbau des Meniskus nach. Die Meniskusoberfläche besteht aus einer ca. $10 \mu \mathrm{m}$ dicken Schicht willkürlich orientierter Fibrillen. Darunter folgt femur- und tibiaseitig eine lamelläre Schicht, in der sich die Kollagenfasern überkreuzen. Diese Schicht ist ca. $150 \mu \mathrm{m}$ dick. Den Hauptbestandteil im Meniskusinneren stellen zirkumferent verlaufende Kollagenfaserbündel dar. Diese werden vereinzelt von radialen Kollagenfasern umschnürt (Abb. 2).

\section{Tabelle 1}

Einteilung des Meniskus basierend auf dem Grad der Vaskularisierung.

\begin{tabular}{|c|c|c|}
\hline Zone & Lokalisation & Kennzeichen \\
\hline rot-rote Zone & außen, kapselnah & vaskularisiert \\
\hline rot-weiße Zone & Übergangszone & $\begin{array}{l}\text { weniger gut } \\
\text { vaskularisiert }\end{array}$ \\
\hline weiß-weiße Zone & innen & avaskulär \\
\hline
\end{tabular}

Die kapselnahen Bereiche des medialen und lateralen Meniskus weisen eine Vaskularisierung auf (Abb. 3) [3,4]. Der innere Bereich des Meniskus ist vollständig avaskulär und wird durch Diffusion ernährt [5]. Folglich teilt man den Meniskus basierend auf dem Grad der Vaskularisierung in 3 Zonen ein (Tab. 1) [3,6].

\section{Verankerungsligamente}

Am anterioren und posterioren Ende jedes Meniskus ist ein deutlicher Übergang zu den faserreichen meniskotibialen Verankerungsligamenten zu erkennen (Abb. 1 u. Abb. 4). Die Geometrie der Ligamente zeigt deutliche Unterschiede, wohingegen die Insertionen am tibialen Knochen anhand von anatomischen Landmarken sehr gut bestimmt werden können [7].

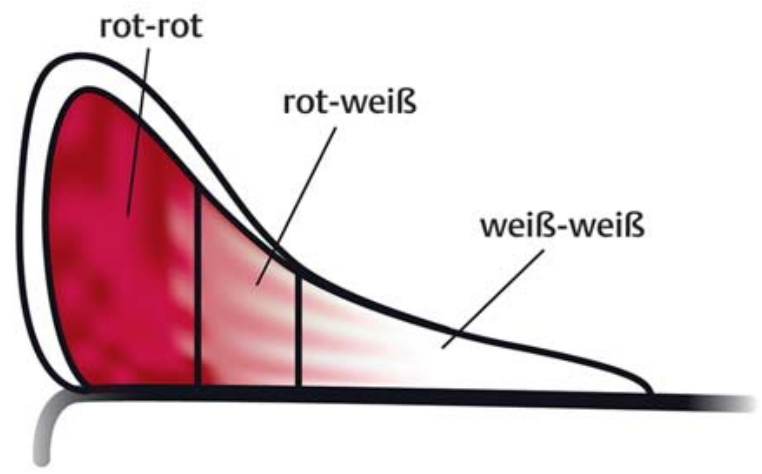

Abb. 3 - Vaskularisierung des Meniskus, welche von außen nach innen abnimmt und nach dem Grad der Vaskularisierung in 3 Zonen unterteilt wird: die rot-rote, rot-weiße, weiß-weiße Zone.

Abb. 2 - Aufbau des Meniskus in 3 Schichten: Oberfläche (1), Lamellenschicht (2) und zentraler Hauptteil (3). 


\section{Übersicht}

Anatomie
- Der Meniskus teilt sich von anterior nach posterior in
Vorderhorn, Pars intermedia und Hinterhorn ein.
- Von der Peripherie ins Innere nimmt die Vaskularisierung
des Meniskus bis zur vollständigen Avaskularität ab.
- Die Menisken des Kniegelenks weisen einen biphasischen
Aufbau aus einer Matrix aus Kollagen-I-Fasern und
Proteoglykanen sowie interstitieller Flüssigkeit auf.
- Der Meniskus besitzt einen dreischichtigen Aufbau mit
unterschiedlicher Anordnung von Kollagenfasern und
-fibrillen.

Anterior inseriert das fächerförmige und flache Verankerungsligament des medialen Meniskus im Bereich der Area intercondylaris ca. $7 \mathrm{~mm}$ anterior der Insertion des vorderen Kreuzbandes [8,9]. Das posteriore Verankerungsligament des medialen Meniskus inseriert im hinteren Bereich der Fossa intercondylaris, anterior der hinteren Kreuzbandinsertion [8-10]. Die Insertionen des medialen Meniskus liegen weiter voneinander entfernt als die der Verankerungsligamente des lateralen Meniskus, wodurch letzterer deutlich mobiler ist. Das anterolaterale Verankerungsligament inseriert anterior des Tuberculum intercondylare laterale und seitlich des vorderen Kreuzbandes [8]. Posterior inseriert das flache und breite Verankerungsligament des lateralen Meniskus im Bereich vom medialen zum lateralen interkondylären Höcker [9].

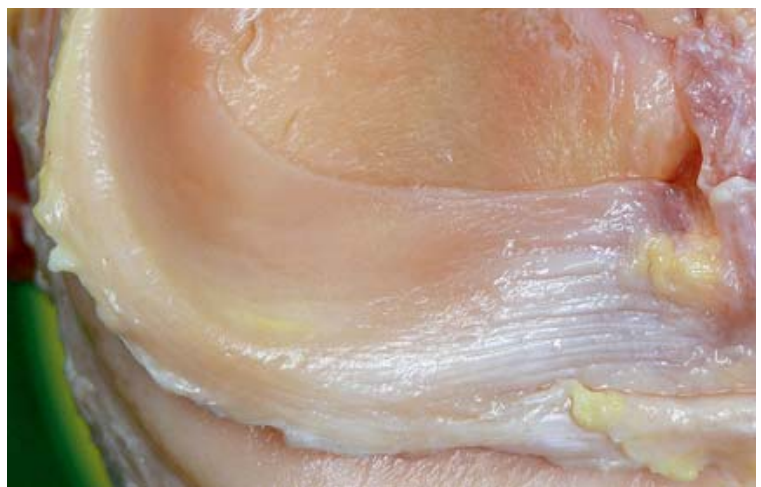

Abb. 4 - Detailaufnahme des anterolateralen Übergangs von Meniskusgewebe zu meniskotibialem Insertionsligament.
Wie der Meniskus besitzen die Verankerungsligamente ebenfalls einen biphasischen Aufbau [11,12], wobei etwa zwei Drittel des Gesamtgewichts auf interstitielle Flüssigkeit entfallen. Die solide Matrix besteht hauptsächlich aus Kollagenfasern Typ I (80\%; [13]), die in den Meniskuskörper übergehen (Abb. 4).

\section{Funktion des Meniskus und seiner Bänder}

- Die Hauptfunktion der keilförmigen Menisken ist die Vergrößerung der kraftübertragenden Kontaktfläche zwischen Femur und Tibia und die daraus resultierende Reduzierung des Kontaktdruckes. Durch ihre Geometrie, Struktur und Verankerung reduzieren sie Spannungsspitzen bei der Lastübertragung und schützen so den Gelenkknorpel vor überlastungsbedingter Degeneration [14-16].

Wenn das Kniegelenk axial belastet wird, werden die Menisken aufgrund ihres keilförmigen Querschnitts aus dem Kniegelenksspalt herausgedrückt. Da sie über ihre Verankerungsligamente im Knochen befestigt sind, entstehen Zugspannungen in Umfangsrichtung der Menisken (Abb. 5; [17]), und eine komplette Extrusion wird verhindert. Dadurch sind die Menisken in der Lage, Druck aufzunehmen und bis zu $81 \%$ der axialen Gelenkkräfte zu übertragen $[18,19]$.

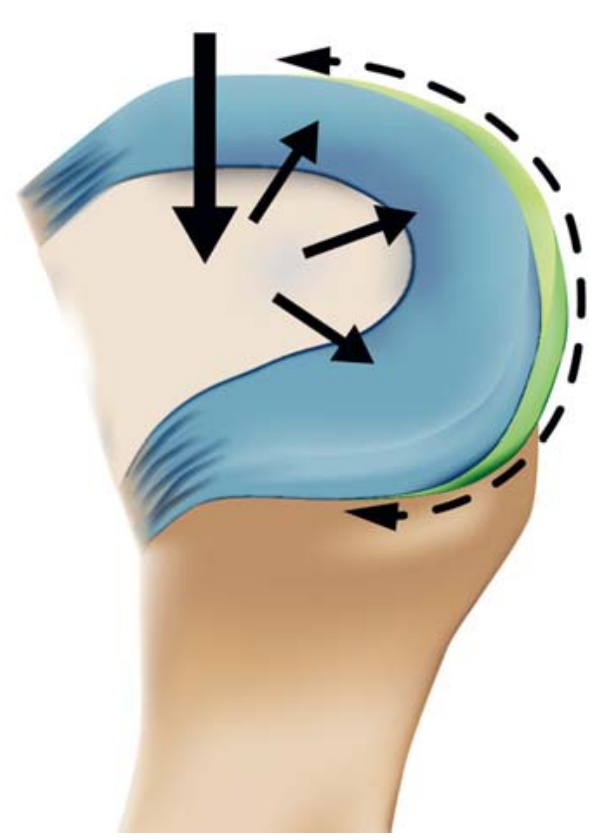

Abb. 5 - Unter Belastung des Kniegelenks hat der keilförmige Meniskus das Bestreben, aus dem Gelenk heraus zu extrudieren. In Umfangsrichtung (gestrichelte Linie) entstehen zirkumferente Spannungen, die über die Insertionsligamente vom Knochen aufgenommen werden. 


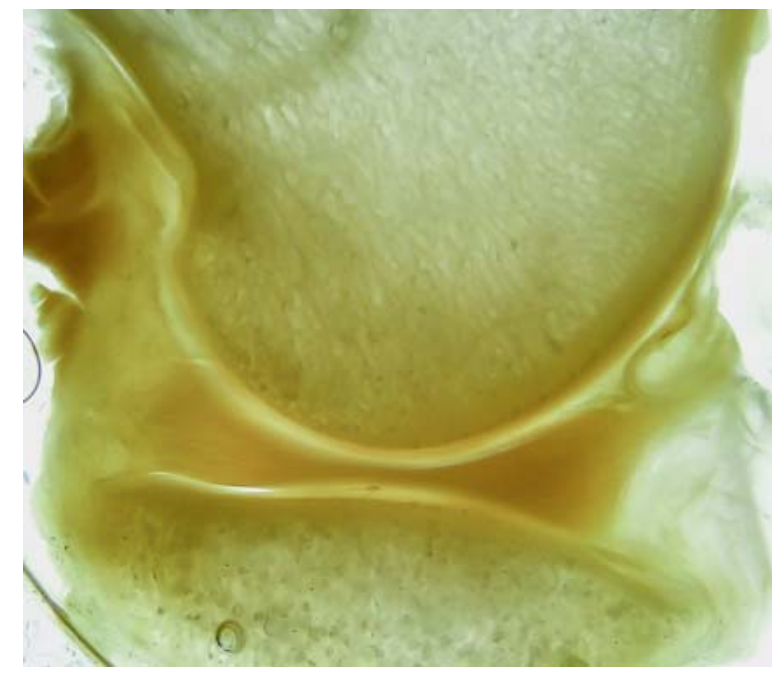

Abb. 6 - Sagittalschnitt durch das mediale Kniegelenkkompartiment eines Schafs. Deutlich ist zu sehen, wie der Meniskus den Raum zwischen der runden Femurkondyle und dem eher flachen Tibiaplateau ausgleicht und so die Kontaktfläche zwischen den artikulierenden Gelenkflächen vergrößert. Hierdurch wird der Druck auf den Gelenkknorpel wesentlich reduziert.

Die Menisken gleichen die Inkongruenz zwischen den Gelenkflächen an distalem Femur und proximaler Tibia aus (Abb. 6). Durch ihre Beweglichkeit, Flexibilität und Form sind sie in der Lage, diese Aufgabe in jeder Flexionsund Rotationsstellung der Tibia zu bewältigen [20].

Entsprechend den unterschiedlichen Roll-Gleit-Bewegungen der beiden Femurkondylen muss der laterale Meniskus größere Bewegungen als der mediale Meniskus vollführen. Um dieser Aufgabe gerecht zu werden, ist der laterale Meniskus an seiner Peripherie nicht wie der mediale Meniskus mit der Kapsel verwachsen, und seine Insertionsbänder liegen enger beieinander. Für die anterioren Hörner des lateralen und medialen Meniskus wurden gegenüber den posterioren Hörnern größere Verschiebungen ermittelt. Während der Flexion des Kniegelenks von $0^{\circ}$ bis $90^{\circ}$ unter Belastung zeigte sich eine mittlere posteriore Verschiebung von 7,1 $\mathrm{mm}$ im anterioren Horn und 3,9 mm im posterioren Horn des medialen Meniskus. Das anteriore Horn des lateralen Meniskus verschiebt sich um 9,5 mm nach posterior und das posteriore Horn um 5,6 mm [21]. Thomson et al. kamen zu ähnlichen Ergebnissen [20].

Zusätzlich zur wichtigsten Funktion der Lastübertragung im Kniegelenk tragen die Menisken zur Gelenkschmierung [22,23], Nährstoffverteilung [22,23] und Propriozeption $[10,24,25]$ bei. Durch seine viskoelastischen Materialeigenschaften spielt der Meniskus potenziell auch eine Rolle als Schockabsorber [26-28]. Dies wird jedoch

\section{Übersicht}

\section{Funktion des Meniskus \\ - Die Hauptfunktion des Meniskus ist die Lastübertragung zwischen Femur und Tibia. \\ - Durch die Fixierung der Menisken an Bändern wird ihre Extrusion aus dem Gelenk unter Last verhindert und so die kraftübertragende Fläche vergrößert, was zur Redu- zierung des Gelenkdruckes führt. \\ - Weitere Funktionen des Meniskus sind die Gelenk- schmierung, Nährstoffverteilung und Propriozeption.}

kontrovers diskutiert, da ein aktuelles Review [29] die Schlussfolgerungen der zuvor zitierten Arbeiten infrage stellt.

\section{Biomechanische Eigenschaften}

\section{Meniskuskörper}

Zur Charakterisierung der biomechanischen Eigenschaften des Meniskus wurden eine Reihe von Zug- und Druckprüfungen an gesunden Menisken durchgeführt. Die Erkenntnisse aus solchen Versuchen sind essenziell für ein besseres Verständnis ihrer Funktionsweise, aber auch für die Neu- und Weiterentwicklung von Meniskusersatzmaterialien.

\section{- Eigenschaften unter Zugbelastung}

Die anisotropen (richtungsabhängigen) Materialeigenschaften machen eine Testung parallel zu den zirkumferent verlaufenden Kollagenfasern (zirkumferente Orientierung) und senkrecht dazu (radiale Orientierung) unabdingbar (Abb. 7). Das Elastizitätsmodul (von der Geometrie unabhängige Steifigkeit) in zirkumferenter Richtung ist etwa eine Größenordnung höher als in radialer Richtung. In zirkumferenter Richtung variiert das Elastizitätsmodul zwischen $85 \mathrm{MPa}$ [30] und $175 \mathrm{MPa}$ [31]. Radiale Proben weisen ein Elastizitätsmodul von ca. $11 \mathrm{MPa}$ auf [32]. Außerdem variiert der Wert in Abhängigkeit von weiteren Faktoren:

- der Lokalisation (Vorderhorn, Pars intermedia, Hinterhorn; [30-32]),

- der Frage, ob es sich um einen medialen oder lateralen Meniskus handelt $[31,32]$ und

- der Schicht (Oberfläche, lamelläre Schicht oder Meniskusinneres; [32]), die getestet wurde. 

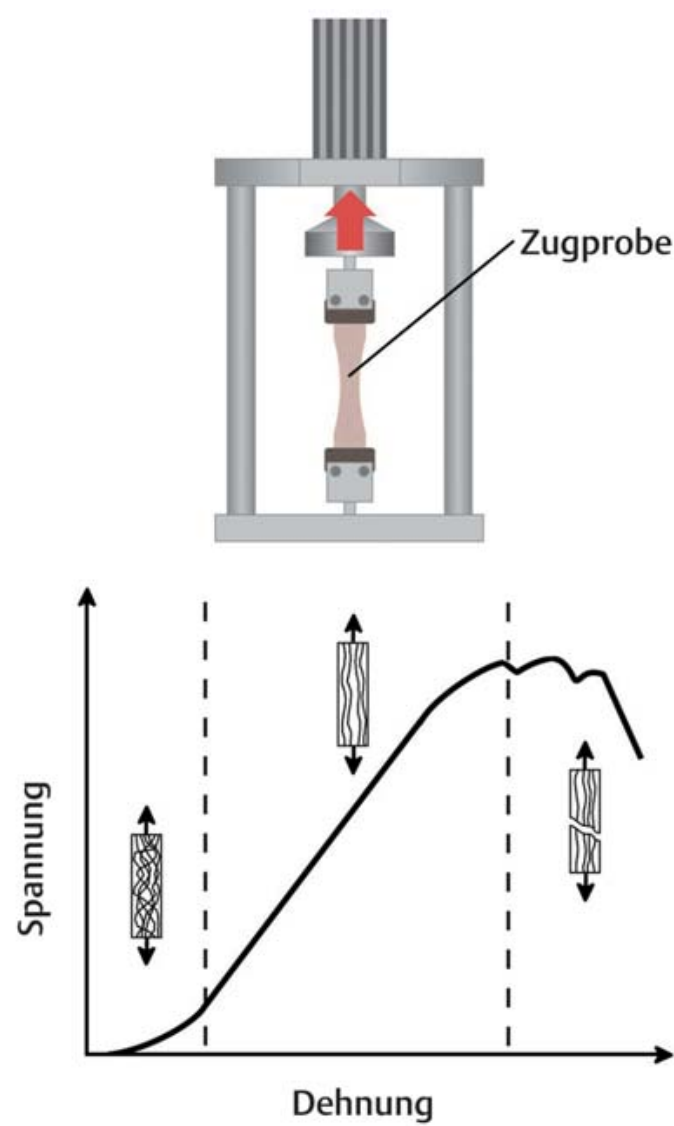

Abb. 7 - Zugversuch an einem Präparat und der dazugehörigen Spannungs-Dehnungs-Kurve mit ihren 3 charakteristischen Abschnitten. Der 1. nicht lineare Abschnitt wird durch die Ausrichtung der Kollagenfasern in Zugrichtung hervorgerufen. Daraufhin folgt ein Abschnitt, in welchem sich die Fasern zunächst linear dehnen, bis die ersten Kollagenfasern reißen und der Verlauf degressiv wird. Anschließend kommt es im 3. Teil der Kurve zum vollständigen Versagen.

\section{- Eigenschaften unter Druckbelastung}

Durch Druckversuche, wie Kriech- (Kraft konstant, Abb. 8) oder Relaxationstest (Verformung konstant), können die viskoelastischen (zeitabhängigen) Eigenschaften des Meniskus charakterisiert werden. Ein Maß für die Durchlässigkeit der porösen Matrix ist die Permeabilität, welche im Meniskus ca. 2,1 × $10^{-15} \mathrm{~m}^{4} / \mathrm{Ns}$ [33-35] beträgt. Die Permeabilität steht hierbei im Zusammenhang mit dem Proteoglykangehalt des Meniskus. Ein erhöhter Proteoglykangehalt führt zu einer erhöhten Wasserbindungsfähigkeit und damit zu einer geringeren Permeabilität. Außerdem kann bei einem Druckversuch nach Erreichen des Gleichgewichtszustandes der sogenannte Aggregate Modulus $\left(\mathrm{H}_{\mathrm{A}}\right)$ bestimmt werden, welcher im Meniskus etwa 0,11 MPa beträgt $[34,35]$ und ein Maß für die Steifigkeit der festen Matrix ist.
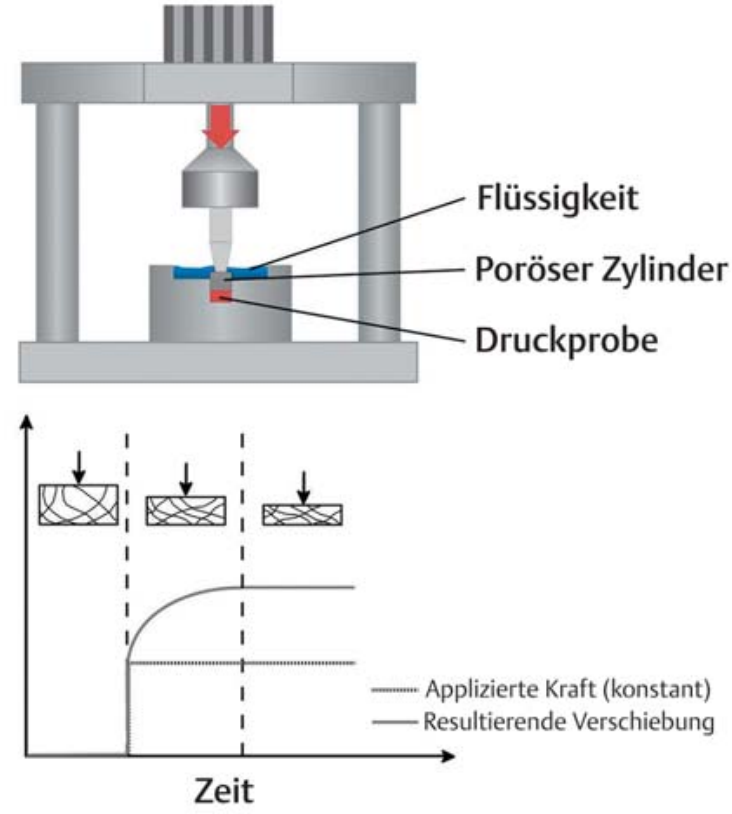

Abb. 8 - Kriechversuch an einer zylinderförmigen Druckprobe. Durch die konstante Belastung wird Flüssigkeit aus dem Präparat gedrückt, was im zeitlichen Verlauf zu einer Zunahme der Verformung bis zum Erreichen eines Gleichgewichtszustandes führt.

Wie auch bei Zugversuchen können die Werte in Abhängigkeit vom medialen und lateralen Meniskus [34] und der Lokalisation im Meniskus (Vorderhorn, Pars intermedia, Hinterhorn; [34,35]) variieren.

\section{Meniskotibiale Bänder}

Zugversuche an den meniskotibialen Verankerungsligamenten haben ergeben, dass die anterioren Verankerungsligamente (169 MPa) ein höheres Elastizitätsmodul (E-Modul) im Vergleich zu den posterioren Verankerungsligamenten (91 MPa) besitzen [12]. Die Steifigkeit der Verankerungsligamente liegt zwischen $102 \mathrm{~N} / \mathrm{mm}$ [36] und $216 \mathrm{~N} / \mathrm{mm}$ [12], was vergleichbar mit der Steifigkeit des vorderen Kreuzbandes ist (129-182 N/mm [37]). Die maximale Belastbarkeit der Bänder liegt zwischen 400 und $650 \mathrm{~N}$, was ein deutliches Indiz für ihre hohe Bedeutung darstellt $[12,36]$.

Allerdings wurden an humanen Kniegelenkpräparaten unter quasistatischen Bewegungs- und Belastungsbedingungen lediglich Kräfte in den meniskotibialen Bändern in Höhe von $25 \mathrm{~N}$ ermittelt, was gemessen an ihrer hohen Reißkraft sehr wenig erscheint [36]. Es ist deshalb zu vermuten, dass es Belastungssituationen geben muss, die die Bänder wesentlich stärker beanspruchen. Um dies zu zeigen, sind weitere experimentelle Untersuchungen, 


\section{Übersicht}

\section{Biomechanische Eigenschaften \\ - Der Meniskus besitzt richtungsabhängige Eigenschaften. In zirkumferenter Richtung ist er im Zugversuch etwa $10 \times$ steifer als in radialer Richtung. \\ - Im Druckversuch können die zeitabhängigen viskoelasti- schen Eigenschaften charakterisiert werden. \\ - Die meniskotibialen Ligamente besitzen eine Reißkraft in Höhe von 400-650 N.}

z.B. unter dynamischen und stoßförmigen Kniegelenkbelastungen, erforderlich.

\section{Funktionsverlust bei Verletzungen}

Meniskusläsionen durch traumatische Ereignisse oder degenerative Prozesse können entsprechend ihrer Form charakterisiert werden (Abb. 9). Basierend auf dem Klassifizierungssystem der International Society of Arthroscopy, Knee Surgery and Orthopaedic Sports Medicine (ISAKOS) werden sie eingeteilt wie in der Infobox „Prinzipien“ dargestellt.

Häufig werden solche Risse mit arthroskopischer partieller Meniskektomie behandelt. Biomechanische Untersuchungen haben gezeigt, dass dies zu einer reduzierten Funktionalität des Meniskus führt und zu negativen Langzeiteffekten wie frühzeitiger Arthrose [14-16]. Die Funktionalität ist hauptsächlich durch die durchtrennten zirkumferenten Kollagenfasern, die die Belastung nicht mehr aufnehmen können, eingeschränkt $[2,38,39]$.

\section{Prinzipien}

Einteilung der Meniskusläsionen gem. International Society of Arthroscopy, Knee Surgery and Orthopaedic Sports Medicine (ISAKOS)

- Longitudinalriss

- Horizontalriss

- Radialriss

- vertikaler Lappenriss

- horizontaler Lappenriss

\section{Partielle Meniskektomie}

Der Funktionsverlust durch Teilmeniskektomie wurde hauptsächlich mit in vitro kontaktmechanischen Untersuchungen zwischen Meniskus und Tibia nachgewiesen. Die intakten Menisken tragen etwa 80\% der gesamten Kniebelastung [18] und decken bei Belastungen zwischen $200 \mathrm{~N}$ und $1000 \mathrm{~N}$ im Mittel 71-82\% der Kontaktfläche zwischen Femur und Tibia ab [40].

D Durch partielle Meniskektomie kommt es zu einer verringerten Kontaktfläche und damit zu erhöhtem Kontaktdruck, was die Lastübertragungsfunktion des Meniskus beeinträchtigt.

Je mehr Meniskusgewebe reseziert wird, desto höher wird der Kontaktdruck. Beispielsweise konnten Lee et al. zeigen, dass im gestreckten Zustand des Beins und einer Belastung von 1,8 kN bei $50 \%$ Resektion der radialen Tiefe des medialen Hinterhorns die Kontaktfläche um $21 \%$ im medialen Kompartiment abnimmt, wohingegen der Kontaktdruck im gleichen Kompartiment um 23\% zunahm [38].

Im Tiermodell wurde der Unterschied zwischen Resektion eines Blattes bei Horizontalriss und Resektion des
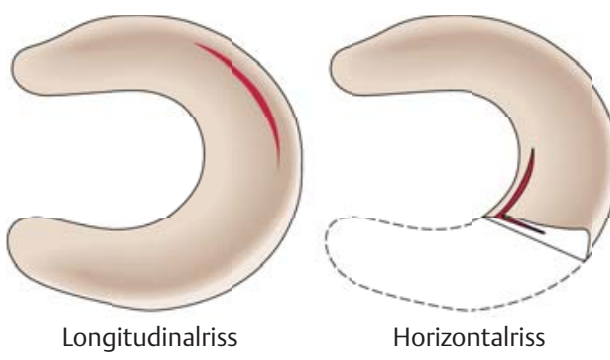

Horizontalriss

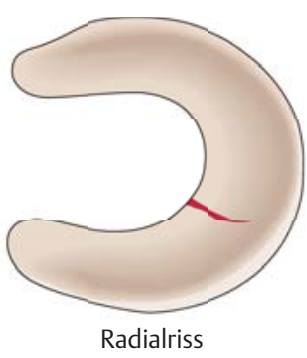

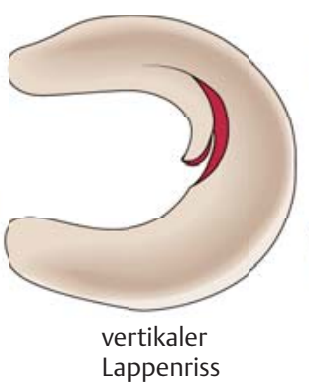

Lappenriss

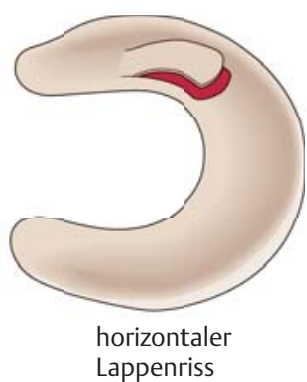

Abb. 9 - Rissformen des Meniskus. 
oberen und unteren Blattes ermittelt. Es konnte gezeigt werden, dass die Kontaktfläche zwischen intaktem und Resektion eines Blattes um 40\% abnimmt und weitere 15\% bei Resektion des zweiten Blattes. Der Spitzenkontaktdruck zwischen Resektion eines und beider Blätter zeigte keinen Unterschied (3\%) [41].

Klinische Studien $[42,43]$ zeigen, dass eine partielle Meniskektomie des lateralen Meniskus eine schlechtere Prognose hat als eine partielle Meniskektomie des medialen Meniskus. Dies kann biomechanisch vermutlich durch eine höhere Beweglichkeit des lateralen Meniskus erklärt werden, bleibt aber zu beweisen.

Ein Komplettriss eines Verankerungsligaments (Root Tear) kommt einer Teil- bis zur Totalmeniskektomie im Hinblick auf den biomechanischen Funktionsverlust gleich. Es konnte gezeigt werden, dass sich der maximale Kontaktdruck in dem geschädigten Kompartiment bei einer Belastung von 1,8 kN um 32\% erhöht und die Kontaktfläche um $20 \%$ verringert [44].

\section{Totale Meniskektomie}

Eine komplette Resektion des hinteren medialen Meniskus, mit Durchtrennung der Randleiste, führt im medialen Kompartiment zu einer Erhöhung des maximalen Kontaktdruckes um bis zu $68 \%$, gleichzeitig nimmt die Kontaktfläche um 50\% im Vergleich zum intakten Zustand ab (Abb. 10) [39]. Der Unterschied zwischen einer Resektion eines kompletten Segments mit Durchtrennung der Randleiste und einer totalen Meniskektomie war verschwindend gering. Der mittlere Kontaktdruck nahm nach totaler Meniskusentfernung im Vergleich zur segmentalen Resektion lediglich um weitere $2 \% \mathrm{zu}$ [38].

\section{Übersicht}

\section{Funktionsverlust bei Verletzungen}

- Partielle Meniskektomien führen zu einer erheblich reduzierten Kontaktfläche und damit zu erhöhtem Kontaktdruck.

- Je mehr Meniskusgewebe reseziert wird, desto höher der Kontaktdruck im Gelenk und das Risiko einer Arthrose.

- Abrisse der meniskotibialen Bänder erhöhen den Kontaktdruck im Kniegelenk um bis zu 32\%. Die Funktion des Meniskus ist dann nicht mehr gewährleistet, da seine komplette Extrusion aus dem Gelenk nicht verhindert wird.

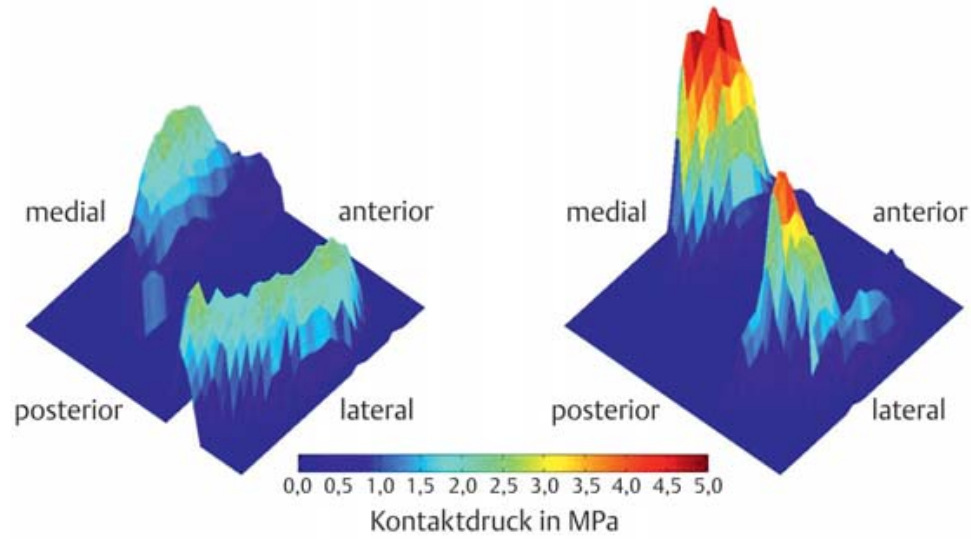

Abb. 10 - Kontaktdruck auf dem Tibiaplateau eines intakten Kniegelenks mit medialem und lateralem Meniskus in $30^{\circ}$-Flexion (links) und eines Kniegelenks nach Totalresektion des medialen Hinterhorns (rechts) jeweils unter einer Belastung von $1 \mathrm{kN}$.

\section{Wiederherstellung der Funktion}

Die Kenntnis der nachteiligen Auswirkungen einer totalen oder partiellen Meniskektomie führten zu dem Bestreben, so wenig Meniskusgewebe wie möglich zu resezieren. Außerdem wurden Nahtverfahren und spezielle Implantate entwickelt, um den verletzten Meniskus vollständig zu erhalten. Es eignen sich allerdings nur bestimmte Meniskusrissformen für die Wiederherstellung. Hierzu gehören insbesondere Risse in der peripheren durchbluteten Zone. Die inneren Anteile des Meniskus sind nicht vaskularisiert und von geringer Zellularität, wodurch sich sein schlechtes regeneratives Potenzial in dieser Zone erklärt.

\section{Naht/Anker}

Um eine Heilung des Meniskus zu ermöglichen, ist es erforderlich, die Rissflächen unter Druck zu adaptieren. Die hierzu verwendeten Nahttechniken und Anker müssen diesen Druck bis zur Heilung des Gewebes aufrechterhalten, ohne dass es zu einem Aufklaffen des Risses unter Bewegung und Belastung des Kniegelenks kommt.

Ein wichtiger biomechanischer Parameter ist deshalb die Ausreißkraft von Meniskusnähten und Ankern.

Hierzu existieren eine Reihe von Arbeiten, die meistens mehrere Verfahren miteinander vergleichen (z.B. [4548]). Es zeigte sich, dass Nähte je nach Nahtstärke und Nahttechnik typischerweise Auszugskräfte zwischen 60 und $120 \mathrm{~N}$ aufweisen. Dabei wiesen stärkere und vertikale Nähte eine höhere Belastbarkeit auf als Nähte mit geringerem Durchmesser und Horizontalnähte. 
Ankerimplantate in Form von resorbierbaren Schrauben, Pfeilen und anderen Formen zeigten meist geringere maximale Haltekräfte zwischen 20 und $60 \mathrm{~N}$ auf. Auch die Steifigkeit der Fixierung variiert und liegt z.B. beim Nahtanker FAST-FIX (Smith \& Nephew) bei ca. $10 \mathrm{~N} / \mathrm{mm}$ und bei verschiedenen Ankerimplantaten bei ca. $5 \mathrm{~N} / \mathrm{mm}$ [49]. Die geringeren Auszugskräfte der Implantate gegenüber den Nahttechniken müssen jedoch nicht unbedingt von Nachteil sein, da gezeigt werden konnte, dass z.B. durch Verwendung von 3 Meniskusschrauben (Auszugskraft je ca. $25 \mathrm{~N}$ ) das Aufklaffen des Rissspaltes auch unter Bewegung und Belastung des Kniegelenks suffizient vermieden werden kann [50].

$\mathrm{Zu}$ beachten ist jedoch bei dieser Art von Implantaten, dass manche bauartbedingt Implantatköpfe aufweisen, die auf der Oberfläche des Meniskus zu liegen kommen und somit in Kontakt zur femoralen Kondyle stehen. Bei Gelenkbewegung unter Belastung kann es hier zu Schädigungen des Gelenkknorpels durch Reibungseffekte kommen. Auch bei Implantaten, die im Meniskusgewebe versenkt werden, kann es zum Herauswandern des Ankers aus dem Meniskus und damit zur Interaktion mit dem Gelenkknorpel kommen.

- Aus diesem Grund sind Nahttechniken im Prinzip den Meniskusankern vorzuziehen.

Da die Meniskusnaht insbesondere im schwer zugänglichen Hinterhornbereich jedoch chirurgisch sehr anspruchsvoll ist, wurden Kombinationen von Naht und Anker entwickelt, bei denen mithilfe eines Implantationstools kleine resorbierbare Nahtanker in rein arthroskopischer Technik hinter die Basis des Meniskus gebracht und mittels vorgeknüpfter Knoten an der Meniskusoberfläche fixiert werden (RapidLoc, DePuy Mitek; FAST-FIX, Smith \& Nephew). Der Nahtanker FAST-Fix hält Ausreißkräften von ca. 80 N stand [49].

Die meisten Studien prüften die Auszugskräfte lediglich direkt nach Naht bzw. Implantation. Dynamische Belastungen, wie sie im Kniegelenk vorkommen, können die Auszugskräfte von Nähten und Ankern jedoch mit der Zeit reduzieren [46]. Dies sollte bei der Wahl der Fixationstechnik beachtet werden.

\section{Ersatzimplantate}

Häufig kommt es zu Rissen im nicht vaskularisierten Bereich des Meniskus (weiß-weiße Zone), die nicht durch Fixierung mit Nähten oder Ankern zur Heilung gebracht werden können. In diesen Fällen kann eine teilweise oder totale Entfernung des Meniskus mit den oben beschrie- benen nachteiligen Auswirkungen auf die Kraftübertragung im Kniegelenk indiziert sein.

Alternativ kommt der Ersatz von Meniskusgewebe durch Implantate infrage. Hierfür existiert inzwischen eine Reihe von Forschungsansätzen mit unterschiedlichen Strategien. Zum einen sind dies regenerative Methoden, bei denen teilweise oder vollständig resorbierbare poröse Scaffolds als Platzhalter in den Meniskusdefekt eingesetzt werden. Hier ist das Ziel, Umbauprozesse in Gang zu setzen, um das ursprüngliche Gewebe wiederherzustellen. Im klinischen Einsatz sind bisher lediglich zwei regenerative Implantate [51]:

- das Collagen Meniscal Implant (CMI, Ivy Sports Medicine), welches aus hoch gereinigtem Kollagen Typ I besteht, und

- das Actifit Implantat (Orteq Sports Medicine), das aus einem Komposit aus schnell degradierenden Polycaprolactonsegmenten und sehr langsam degradierenden Urethananteilen besteht.

Klinisch werden für beide Implantate $z$.T. gute Resultate mit Schmerzlinderung beschrieben, allerdings gibt es lediglich wenige prospektive Studien mit Kontrollgruppen, die einen Mehrwert des Implantats gegenüber einer Teilmeniskektomie wissenschaftlich nachweisen.

Die biomechanischen Eigenschaften beider Materialien sind zum Implantationszeitpunkt zunächst unzureichend. Ob nach Ab- bzw. Umbau des Materials ein Gewebe mit dem meniskusähnlichen biomechanischen Materialeigenschaften entsteht, ist bisher nicht nachgewiesen.

Eine interessante Idee ist die Verwendung von dezellularisierten humanen Menisken als Gerüst für das Tissue Engineering des Meniskus. Sandmann et al. konnten zeigen, dass sowohl Steifigkeit als auch Restkraft des dezellularisierten Gerüstes unter zyklischen Belastungen sich nicht von denen nativer Menisken unterschieden, was eine gute Voraussetzung für einen Meniskusersatz ist [52]. Jedoch muss auch für dieses Material erst in vivo nachgewiesen werden, dass es die Bildung eines biomechanisch suffizienten biologischen Gewebes unterstützt.

Außer regenerativen Ansätzen gibt es auch noch Ideen, Meniskusgewebe dauerhaft durch ein Implantat zu ersetzen. Mit permanenten Implantaten ist es zurzeit noch eher möglich, adäquate mechanische Eigenschaften zu erzeugen als mit regenerativen Methoden. Polycarbonat [53], Seidenfibroin [54] und andere Polymere [51] wer- 
Übersicht

\section{Wiederherstellung der Funktion}

- Der Teil- oder Totalersatz des Meniskus ist noch keine weitläufig etablierte Methode. Häufig fehlen prospektiv randomisierte Studien mit Kontrollgruppen, die nachweisen könnten, ob die zurzeit kommerziell erhältlichen Implantate einen Mehrwert im Vergleich zur Teilmeniskektomie darstellen.

- Als Totalersatz können Allograft-Transplantate verwendet werden, bei denen die Fixierung von hoher Bedeutung für die biomechanische Funktion ist.

- Alle anderen in der Literatur beschriebenen Ansätze zu regenerativen Methoden oder Permanentimplantaten sind noch in der Entwicklungsphase.

den verwendet, um den Meniskus teilweise oder ganz zu ersetzen. Allerdings haben diese Implantate noch nicht den Weg in die Klinik gefunden oder wurden nur in sehr kleinen Serien klinisch getestet.

Klinisch eingesetzt werden hingegen an einigen Zentren Meniskustransplantate von humanen Spendern (Allograft). Während mehrere Langzeitstudien von durchaus positiven Ergebnissen berichten [55-57], ist zu beachten, dass die Fixierungstechnik beim Allograft von großer Bedeutung ist. Wie oben beschrieben nehmen die nativen meniskotibialen Bänder die zirkumferenten Spannungen im Meniskus auf und verhindern dadurch ein Extrudieren. Dies gilt natürlich auch für den transplantierten Meniskus. So führt eine Fixierung eines Allografts mit transtibialen Nähten zu einer vermehrten Extrusion und so zwangsläufig zu einer verminderten biomechanischen Funktion des Meniskus im Vergleich zur rigideren Fixation mit Knochenblöcken [58].

\section{Zusammenfassung}

Die Hauptfunktion der knorpeligen Menisken des Kniegelenks ist die Reduzierung des Druckes auf den femoralen und tibialen Gelenkflächen. Meniskusverletzungen machen häufig Teil- oder in seltenen Fällen auch die Totalresektion eines Meniskus erforderlich, was zur signifikanten Erhöhung des Kontaktdruckes und in der Folge nicht selten zu Gelenkarthrose führt.
Die biomechanische Funktion der Menisken wird durch ihre spezielle Anatomie und ihren ultrastrukturellen Aufbau gewährleistet. Neben den biomechanischen Eigenschaften der Menisken selbst kommt auch den meniskotibialen Bändern, die Reißkräfte von 400-650 N besitzen, eine entscheidende Bedeutung zu.

Nur durch die rigide Befestigung der Menisken am Knochen ist die Erzeugung von zirkumferenten Spannungen in den Menisken möglich, die eine komplette Extrusion aus dem Gelenk verhindern und so eine Vergrößerung der lastübertragenden Fläche bewirken. Schon die Resektion von Teilen eines Meniskus kann den Kontaktdruck stark erhöhen, und Komplettrisse im Hornbereich oder eines Verankerungsligamentes führen zu einem Anstieg des Kontaktdruckes um über 30\%. Deshalb ist eine möglichst sparsame Resektion wichtig, um den Funktionsverlust der Menisken möglichst gering zu halten.

Liegt ein Riss in der vaskularisierten Zone (rote Zone), ist eine Heilung nach Stabilisierung einer Läsion mittels Naht oder Anker möglich. Dabei erleichtern spezielle Nahtanker die operative Technik der ansonsten sehr anspruchsvollen Naht insbesondere im Hinterhornbereich.

Meniskusersatz bzw. Teilersatz stellen noch keine allgemein akzeptierte Option dar. Zwar existieren zwei Ersatzgerüste auf dem Markt mit z.T. positiven klinischen Ergebnissen, jedoch fehlen häufig noch prospektive Studien mit Kontrollgruppen, um deren tatsächlichen Nutzen nachzuweisen. Weitere regenerative Ansätze zum Meniskusersatz und Permanentimplantate sind in der Entwicklung, und man kann gespannt sein, was die nächsten Jahre in der Forschung bringen werden.

Interessenkonflikt: Die Autoren geben an, dass eine Kooperation mit Fa. Orthox Ltd., Abingdon, UK, zur Entwicklung und Testung eines Meniskusersatzimplantats besteht. 


\section{Quellenangaben}

1 Mow VC, Huiskes R. Basic orthopaedic Biomechanics and MechanoBiology. 3rd ed. Philadelphia, PA: Lippincott Williams \& Wilkins; 2005

2 Petersen W, Tillmann B. Funktionelle Anatomie der Menisken des Kniegelenks Kollagenfasertextur und Biomechanik. Arthroskopie 1998; 11: $133-135$

3 Arnoczky SP, Warren RF. Microvasculature of the human meniscus. Am J Sports Med 1982; 10: 90-95

4 Danzig L, Resnick D, Gonsalves M et al. Blood-supply to the normal and abnormal menisci of the human knee. Clin Orthop Related Res 1983; (172): 271-276

5 Petersen W, Tillmann B. Structure and vascularization of the knee joint menisci. Z Orthop Grenzgebiete 1999; 137: 31-37

6 McDermott ID, Masouros SD, Amis AA. Biomechanics of the menisci of the knee. Curr Orthop 2008; 22: 193-201

7 Johannsen AM, Civitarese DM, Padalecki JR et al. Qualitative and quantitative anatomic analysis of the posterior root attachments of the medial and lateral menisci. Am J Sports Med 2012; 40: 2342 2347

8 Johnson DL, Swenson TM, Livesay GA et al. Insertion-site anatomy of the human menisci: gross, arthroscopic, and topographical anatomy as a basis for meniscal transplantation. Arthroscopy 1995; 11: 386 394

9 Kohn D, Moreno B. Meniscus insertion anatomy as a basis for meniscus replacement: a morphological cadaveric study. Arthroscopy 1995; 11: $96-103$

10 Messner K, Gao J. The menisci of the knee joint. Anatomical and functional characteristics, and a rationale for clinical treatment. J Anat 1998; 193 (Pt 2): 161-178

11 Abraham AC, Moyer JT, Villegas DF et al. Hyperelastic properties of human meniscal attachments. J Biomech 2011; 44: 413-418

12 Hauch KN, Villegas DF, Haut Donahue TL. Geometry, time-dependent and failure properties of human meniscal attachments. J Biomech 2010; 43: 463-468

13 Villegas DF, Donahue TLH. Collagen morphology in human meniscal attachments: A SEM study. Connective Tissue Res 2010; 51: 327 336

14 Higuchi H, Kimura M, Shirakura K et al. Factors affecting long-term results after arthroscopic partial meniscectomy. Clin Orthop Relat Res 2000; 377: $161-168$

15 Petty CA, Lubowitz JH. Does arthroscopic partial meniscectomy result in knee osteoarthritis? A systematic review with a minimum of 8 years' follow-up. Arthroscopy 2011; 27: 419-424

16 Rangger C, Klestil T, Gloetzer W et al. Osteoarthritis after arthroscopic partial meniscectomy. Am J Sports Med 1995; 23: 240-244

17 Masouros SD, McDermott ID, Amis AA et al. Biomechanics of the meniscus-meniscal ligament construct of the knee. Knee Surg Sports Traumatol Arthroscop 2008; 16: 1121-1132

18 Pena E, Calvo B, Martinez MA et al. Finite element analysis of the effect of meniscal tears and meniscectomies on human knee biomechanics. Clin Biomech (Bristol, Avon) 2005; 20: 498-507

19 Walker PS, Erkman MJ. The role of the menisci in force transmission across the knee. Clin Orthop Related Res 1975; 109: 184-192

20 Thompson WO, Thaete FL, Fu FH et al. Tibial meniscal dynamics using three-dimensional reconstruction of magnetic resonance images. Am J Sports Med 1991; 19: 210-216

21 Vedi V, Williams A, Tennant SJ et al. Meniscal movement. An in-vivo study using dynamic MRI. J Bone Joint Surg Br 1999; 81: 37-41
22 Danzig LA, Hargens AR, Gershuni DH et al. Increased transsynovial transport with continuous passive motion. J Orthop Res 1987; 5: 409-413

23 Renström P, Johnson RJ. Anatomy and biomechanics of the menisci. Clin Sports Med 1990; 9: 523-538

24 Day B, Mackenzie WG, Shim SS et al. The vascular and nerve supply of the human meniscus. Arthroscopy 1985; 1: 58-62

25 Gray JC. Neural and vascular anatomy of the menisci of the human knee. J Sports Orthop Sports Phys Ther 1999; 29: 23-30

26 Krause WR, Pope MH, Johnson RJ et al. Mechanical changes in the knee after meniscectomy. J Bone Joint Surg Am Vol 1976; 58: 599 604

27 Kurosawa H, Fukubayashi T, Nakajima H. Load-bearing mode of the knee joint: physical behavior of the knee joint with or without menisci. Clin Orthop Related Res 1980; (149): 283 - 290

28 Voloshin AS, Wosk J. Shock absorption of meniscectomized and painful knees: a comparative in vivo study. J Biomech Eng 1983; 5 : 157-161

29 Andrews S, Shrive N, Ronsky J. The shocking truth about meniscus. J Biomech 2011; $44: 2737-2740$

30 Lechner K, Hull ML, Howell SM. Is the circumferential tensile modulus within a human medial meniscus affected by the test sample location and cross-sectional area? J Orthop Res 2000; 18: 945- 951

31 Fithian DC, Kelly MA, Mow VC. Material properties and structurefunction relationships in the menisci. Clin Orthop Related Res 1990; (252): 19-31

32 Tissakht M, Ahmed AM. Tensile stress-strain characteristics of the human meniscal material. J Biomech 1995; 28: 411-422

33 Joshi MD, Suh JK, Marui T et al. Interspecies variation of compressive biomechanical properties of the meniscus. J Biomed Mater Res 1995; 29: $823-828$

34 Seitz AM, Galbusera F, Krais C et al. Stress-relaxation response of human menisci under confined compression conditions. J Mech Behav Biomed Mater 2013; 26: 68-80

35 Sweigart MA, Zhu CF, Burt DM et al. Intraspecies and interspecies comparison of the compressive properties of the medial meniscus. Ann Biomed Eng 2004; 32: 1569-1579

36 Seitz A, Kasisari R, Claes L et al. Forces acting on the anterior meniscotibial ligaments. Knee Surg Sports Traumatol Arthrosc 2012; 20: $1488-1495$

37 Noyes FR, Grood ES. The strength of the anterior cruciate ligament in humans and Rhesus monkeys. J Bone Joint Surg Am 1976; 58 : 1074-1082

38 Lee SJ, Aadalen KJ, Malaviya P et al. Tibiofemoral contact mechanics after serial medial meniscectomies in the human cadaveric knee. Am J Sports Med 2006; 34: 1334-1344

39 Seitz AM, Lubomierski A, Friemert B et al. Effect of partial meniscectomy at the medial posterior horn on tibiofemoral contact mechanics and meniscal hoop strains in human knees. J Orthop Res 2012; 30 : 934-942

40 Fukubayashi T, Kurosawa $\mathrm{H}$. The contact area and pressure distribution pattern of the knee. A study of normal and osteoarthrotic knee joints. Acta Orthop Scand 1980; 51: 871-879

41 Haemer JM, Wang MJ, Carter DR et al. Benefit of single-leaf resection for horizontal meniscus tear. Clin Orthop Related Res 2007; 457: 194-202

42 Chatain F, Adeleine P, Chambat P et al. A comparative study of medial versus lateral arthroscopic partial meniscectomy on stable knees: 10year minimum follow-up. Arthroscopy 2003; 19: 842-849 
43 Simpson DA, Thomas NP, Aichroth PM. Open and closed meniscectomy. A comparative analysis. J Bone Joint Surg Br 1986; 68: 301 304

44 Marzo JM, Gurske-DePerio J. Effects of medial meniscus posterior horn avulsion and repair on tibiofemoral contact area and peak contact pressure with clinical implications. Am J Sports Med 2009; 37 : $124-129$

45 Kurzweil PR, Friedman MJ. Meniscus: Resection, repair, and replacement. Arthroscopy 2002; 18 (2 Suppl 1): 33-39

46 Dürselen L, Schneider J, Galler M et al. Cyclic joint loading can affect the initial stability of meniscal fixation implants. Clin Biomech 2003; 18: $44-49$

47 Seil R, Rupp S, Jurecka C et al. Der Einfluss verschiedener Nahtstärken auf das Verhalten von Meniskusnähten unter zyklischer Zugbelastung. Unfallchirurg 2001; 104: $392-398$

48 Brucker PU, Favre P, Puskas G] et al. Tensile and shear loading stability of all-inside meniscal repairs: an in vitro biomechanical evaluation. Am J Sports Med 2010; 38: 1838-1844

49 Brucker PU, Favre P, Puskas GJ et al. Influence of test temperature on biomechanical properties of all-inside meniscal repair devices and inside-out meniscus sutures-evaluation of an isolated distraction loading, worst-case scenario. Clin Biomech (Bristol, Avon) 2011; 26: 749-753

50 Dürselen L, Hebisch A, Wagner D et al. Meniscal screw fixation provides sufficient stability to prevent tears from gapping. Clin Biomech 2007; 22: 93-99

51 Vrancken AC, Buma P, van Tienen TG. Synthetic meniscus replacement: a review. Int Orthop 2013; 37: 291-299

52 Sandmann GH, Eichhorn S, Vogt S et al. Generation and characterization of a human acellular meniscus scaffold for tissue engineering. J Biomed Mater Res A 2009; 91: 567-574

53 Zur G, Linder-Ganz E, Elsner JJ et al. Chondroprotective effects of a polycarbonate-urethane meniscal implant: histopathological results in a sheep model. Knee Surg Sports Traumatol Arthrosc 2011; 19: 255-263

54 Gruchenberg K, Ignatius A, Friemert B et al. In vivo performance of a novel silk fibroin scaffold for partial meniscal replacement in a sheep model. Knee Surg Sports Traumatol Arthrosc 2014; DOI 10.1007./ s00167-014-3009-2

55 Kazi HA, Abdel-Rahman W, Brady PA et al. Meniscal allograft with or without osteotomy: a 15-year follow-up study. Knee Surg Sports Traumatol Arthrosc 2015; 23: 303-309

56 Kempshall PJ, Parkinson B, Thomas M et al. Outcome of meniscal allograft transplantation related to articular cartilage status: advanced chondral damage should not be a contraindication. Knee Surg Sports Traumatol Arthroscn 2015; 23: 280-289

57 Samitier G, Alentorn-Geli E, Taylor DC et al. Meniscal allograft transplantation. Part 1: systematic review of graft biology, graft shrinkage, graft extrusion, graft sizing, and graft fixation. Knee Surg Sports Traumatol Arthrosc 2015; 23: 310-322

58 Abat F, Gelber PE, Erquicia Jl et al. Suture-only fixation technique leads to a higher degree of extrusion than bony fixation in meniscal allograft transplantation. Am J Sports Med 2012; 40: 1591-1596

\section{Zum Weiterlesen und Vertiefen}

1 Andrews S, Shrive N, Ronsky J. The shocking truth about meniscus. J Biomech 2011; 44: 2737-2740

2 Arnoczky SP, Warren RF. Microvasculature of the human meniscus. Am J Sports Med 1982; 10: 90-95

3 Beaufils P, Verdonk R. The Meniscus. Heidelberg: Springer; 2010

4 Fithian DC, Kelly MA, Mow VC. Material properties and structurefunction relationships in the menisci. Clin Orthop Related Res 1990; 252: $19-31$

5 Lee SJ, Aadalen KJ, Malaviya P et al. Tibiofemoral contact mechanics after serial medial meniscectomies in the human cadaveric knee. Am J Sports Med 2006; 34: 1334-1344

6 Masouros SD, McDermott ID, Amis AA et al. Biomechanics of the meniscus-meniscal ligament construct of the knee. Knee Surg Sports Traumatol Arthrosc 2008; 16: 1121-1132

7 McDermott ID, Masouros SD, Amis AA. Biomechanics of the menisci of the knee. Current Orthop 2008; 22: 193-201

8 Mow VC, Huiskes R. Basic orthopaedic Biomechanics and MechanoBiology. 3rd ed. Philadelphia, PA: Lippincott Williams \& Wilkins; 2005

9 Petersen W, Tillmann B. Funktionelle Anatomie der Menisken des Kniegelenks Kollagenfasertextur und Biomechanik. Arthroskopie 1998; 11: $133-135$

10 Vedi V, Williams A, Tennant SJ et al. Meniscal movement. An in-vivo study using dynamic MRI. J Bone Joint Surg Br 1999; 81: 37-41

11 Vrancken AC, Buma P, van Tienen TG. Synthetic meniscus replacement: a review. Int Orthop 2013; 37: 291-299

\section{Korrespondenzadresse}

Prof. Dr. Lutz Dürselen

Institut für Unfallchirurgische Forschung und Biomechanik

Zentrum für Muskuloskelettale Forschung Ulm (ZMFU)

Universitätsklinikum Ulm

Helmholtzstraße 14

$89081 \mathrm{Ulm}$

Telefon: $0731 / 50055301$

Fax: $\quad 0731 / 50055302$

E-Mail: lutz.duerselen@uni-ulm.de 


\section{Beckengürtel und untere Extremität}

\section{CME Fragen}

\section{CME•thieme.de}

\section{CME-Teilnahme}

- Viel Erfolg bei Ihrer CME-Teilnahme unter http://cme.thieme.de

- Bitte informieren Sie sich vorab online über die Gültigkeitsdauer.

- Sollten Sie Fragen zur Online-Teilnahme haben, unter http://cme.thieme.de/hilfe finden Sie eine ausführliche Anleitung.

Welche Form weist der Meniskus auf?
A rund
B halbmondförmig mit keilförmigen Querschnitt
C halbrund mit rundem Querschnitt
D ringförmig mit keilförmigen Querschnitt
E halbmondförmig mit rechteckigem Querschnitt

Wie hoch ist der Wassergehalt des Meniskus?
A $40-50 \%$
B $50-55 \%$
C $55-60 \%$
D $60-70 \%$
E $70-80 \%$

Was ist die Hauptfunktion des Meniskus?
A Schockabsorption
B Nährstoffversorgung
C Propriozeption
D Lastübertragung zwischen Femur und Tibia
E Gelenkschmierung

Die Lastübertragung im Gelenk durch den Meniskus funktioniert, weil ...
A er an Ligamenten aufgehängt ist und dadurch eine komplette Extrusion des Meniskus aus dem Gelenk verhindert wird.

B er eine hohe Druckfestigkeit hat.

C er eine hohe Zugfestigkeit hat.

D er sich an den Gelenkflächen von Femur und Tibia festsaugt.

E er in zirkumferenter Richtung ca. $8 \times$ steifer ist als in radialer Richtung.
Welche Materialeigenschaft weist der Meniskus nicht auf?
A viskoelastisch
B homogen
C anisotrop
D biphasisch
E nicht linear 
Eine der folgenden Aussagen zu den meniskotibialen Ligamenten ist falsch. Welche?
A Die meniskotibialen Ligamente versagen bei 400-650 N.

B Die Kräfte in den meniskotibialen Ligamenten übersteigen unter quasistatischen Bedingungen kaum $25 \mathrm{~N}$.

C Das E-Modul der anterioren meniskotibialen Ligamente ist fast doppelt so hoch wie das der posterioren.

D Die meniskotibialen Ligamente unterstützen unter axialer Gelenkbelastung die Extrusion der Menisken aus dem Gelenk.

E Die Fixierung des Meniskus am Knochen ist essenziell für seine Funktion.
Was sind die Folgen einer partiellen Meniskektomie?
A Abnahme der Kontaktfläche, Abnahme des Kontaktdruckes

B gleichbleibender Kontaktdruck, Abnahme Kontaktfläche

C Anstieg des Kontaktdruckes, Abnahme der Kontaktfläche

D Anstieg des Kontaktdruckes, Anstieg der Kontaktfläche

E gleichbleibende Kontaktfläche, Anstieg des Kontaktdruckes
Die totale Meniskektomie...

Welche Aussage bezüglich Meniskusreparaturverfahren ist richtig?
A hat keinen Einfluss auf die Kontaktfläche im Kniegelenk.

B erhöht den maximalen Gelenkflächendruck um über $60 \%$.

C ist ein anzustrebender operativer Eingriff bei Meniskusläsionen, um eine weitere Gelenkdestruktion zu verhindern.

D ist ein anzustrebender operativer Eingriff bei Meniskusläsionen, um den Freizeitsportler möglichst schnell zu mobilisieren.

E erhöht den Gelenkflächendruck wesentlich im Vergleich zu einem Hornabriss.
Wodurch ist der Meniskusersatz gekennzeichnet?
A Ankerimplantate haben höhere Ausreißkräfte als Nähte.

B Die Meniskusnaht ist einfacher zu bewerkstelligen als die Implantation eines Ankers.

C Nahtanker halten Belastungen von ca. $80 \mathrm{~N}$ stand.

D Ankerimplantate können das Aufklaffen eines Meniskusrisses nicht verhindern.

E Ankerimplantate halten $150 \mathrm{~N}$ Zugkraft stand.
A Der regenerative Ersatz des Meniskus ist eine etablierte Methode.

B Die Fixierung eines Allograft-Transplantats ist für seine Funktion unerheblich.

c Kommerziell erhältliche Meniskusgerüste weisen eine hohe biomechanische Festigkeit auf.

D Polycarbonat ist ein resorbierbares Material für den Meniskusersatz.

E Mit permanenten Implantaten ist es derzeit eher möglich, adäquate mechanische Eigenschaften zu erzeugen als mit regenerativen Methoden. 